\title{
Environmental factors and the epidemics of COVID-19
}

\author{
Lotfi Aleya $^{1} \cdot$ Weikuan $\mathrm{Gu}^{2} \cdot$ Scott Howard ${ }^{3}$ \\ Published online: 27 July 2021 \\ (C) The Author(s), under exclusive licence to Springer-Verlag GmbH Germany, part of Springer Nature 2021
}

The occurrence, development, and prevention of COVID-19 illustrate the environmental impact of human diseases. Human activities have dramatically impacted earth's environment. Modern human society has increased health and longevity, but has created the conditions for emergence of new pathogens and their rapid spread across the globe (El-Sayed et al. 2021). Several recent publications have proved the close connection between the environment and the COVID-19 pandemic from different perspectives, including the impact of human activities.

Population density, a topic that was rarely mentioned before, is directly linked to the spread of the COVID-19, which is transmitted from person to person, especially in close quarters. Wuhan city is densely populated and its citizens and visitors travel around the world, so it is not surprising that SARS-CoV2 rapidly spread within and beyond the city to affect millions of people worldwide. By analysis of the data from cities in China and in the USA, Yin et al. (2021) reported that population density is positively associated with the percentage of patients with COVID-19 infection, but not influenced by the timing of the first case of COVID-19 in each region.

While human population density and activities are directly related to the transmission of SARS-CoV-2, the relation

Responsible Editor: Philippe Garrigues

Lotfi Aleya

lotfi.aleya@univ-fcomte.fr

Weikuan Gu

wgu@uthsc.edu

Scott Howard

showard5@uthsc.edu

1 Chrono-Environnement Laboratory, UMR CNRS 6249, Bourgogne Franche-Comté University, F-25030 Besançon Cedex, France

2 College of Medicine, University of Tennessee Health Science Center, Memphis, TN 38163, USA

3 College of Nursing, University of Tennessee Health Science Center, Memphis, TN 38105, USA between air pollution caused by humans and the spread of COVID-19 seems to be more complicated. Borisova and Komisarenko (2020) argue that the interaction of the SARS$\mathrm{CoV}-2$ envelope with air pollution particulate matter is possible in humid areas. They pointed out that "Particulate matter can aggravate neurological symptoms of SARS-CoV-2 and vice versa, due to their identical nose-to-brain delivery mechanism and possible interference with neuronal effects." Similarly, Sahoo and colleagues (Sahoo 2021) in India identified correlated air pollutants and meteorological factors with COVID-19 infection.

Indeed, the effect of meteorological factors on the spread of COVID-19 is complex. While the general perception is that higher temperatures make virus less transmissible or less contagious, Raza and colleagues reported that COVID-19 cases and temperature showed a positive correlation, that the correlation between rainfall and COVID-19 cases was negative, and that that the increase in humidity was beneficial to stop the transmission of COVID-19 in Pakistan (Raza et al. 2020a, b). A similar study in Jordan indicated that the weather parameters that best predicted active cases of COVID-19 in the initial wave of transmission was the average daily solar radiation and the weather parameter most predictive of active cases of COVID-19 was the maximum temperature (Abdelhafez et al. 2021). In addition, their study showed that wind speed has a direct relationship with active cases. It seems that COVID-19 has its own favorite set of weather conditions, which are usually not met in one place for all. Furthermore, sampling is important for the analysis of environmental factors, considering the complex interactions of multiple factors (Borges et al. 2021).

If someone says that COVID-19 has changed everything in human life, we would agree. COVID-19 not only spreads to every corner of the earth, but also affects all aspects of our lives. Daily life, travel, and even living habits are all affected. Even smoking habits have been impacted by the requirements to wear masks and maintain social distancing (Kalan et al. 2020).

One of the most embarrassing things for scientists in the study of the impact of the COVID-19 pandemic in each 
country and region has been the prediction of the numbers of infections, deaths, and waves of increased incidence. Repeated waves of COVID-19, each exceeding the previous levels of infection, have forced many forecasting models to modify their predicted numbers time and time again. Of course, many models have accounted for repeated waves and have improved their predictions, which have benefited the measures that governments and the public can implement to reduce morbidity and mortality (El Aferni et al. 2020). In particular, new technology and machine learning approaches to predict the spread of the COVID-19 may have improved predictive capabilities (Malki et al. 2021). Nevertheless, the final prediction of these models needs to be confirmed at the end of the pandemic.

Unfortunately, the work done by most countries to prevent and control the COVID-19 pandemic has fallen short. Reasons include the unprecedented scale of the COVID-19 pandemic, the high degree of infectiousness of SARS-CoV2 and its rapid mutation rate, and its diverse means of transmission. However, research on prevention and control have led to public health interventions including maintaining social distancing, preventive e-guidelines and other educational tools, behavioral control on risk aversion, and surgical masks and sanitizers (Tabish et al. 2020; Raza et al. 2020a, b). Study on the active disinfection using UVC light indicated that a mobile robot equipped with eight UVC lamps can kill $99,999 \%$ of bacteria (Guettari et al. 2020), but its effect on COVID-19 remains to be proven.

To permanently control the pandemic, universal vaccination against all known variants, capacity to rapidly create vaccines for new variants, and discovery and wide dissemination of effective antiviral medicines will all be needed. For example, the potential therapeutic application of hydroxychloroquine (HCQ) in COVID-19 and its impact on the environment have been reviewed (Kumar et al. 2021). Kumar and colleagues indicated that as acidification is vital for the viral entry as well as endosome growth and function, HCQ seems to be crucial to the inhibition of the viral entry to the cells. However, authors noted that its impact on the endosomal/lysosomal morphology and $\mathrm{pH}$ value is still under investigation. Fortunately, no environmental concerns about the use of HCQ have been identified so far (Kumar et al. 2021). Abd Elkodous et al. (2020) proposed the role of nanomaterials in controlling and treating COVID-19 through their antiviral and antibacterial potential with suggested action mechanisms indicating the capability of interaction between these nanomaterials and SARS-CoV-2. The authors suggested that these nanomaterials might be among the possible and most effective cures against coronavirus (Abd Elkodous et al. 2020). Optimal disinfection strategies will complement any medical interventions confirmed to be effective.

In summary, COVID-19 has affected every corner of the world, and many environmental factors may impact the spread and pandemic scale of COVID-19. The approval and widespread use of anti-COVID-19 vaccines have not yet ended the pandemic. With the emergence of different variants of the SARS-CoV-2, COVID-19 will likely coexist with humans for a long time, just like influenza. In the battle against COVID-19, humankind has achieved some initial victories with vaccines and therapeutics, but continued efforts are needed to full eradicate this new threat to humanity at the individual, environmental, and global levels.

\section{References}

Abd Elkodous M, El-Sayyad GS, Abdel-Daim MM (2020 Oct) Engineered nanomaterials as fighters against SARS-CoV-2: the way to control and treat pandemics. Environ Sci Pollut Res Int 17: 1-7. https://doi.org/10.1007/s11356-020-11032-3

Abdelhafez E, Dabbour L, Hamdan M (2021 Jan) The effect of weather data on the spread of covid-19 in Jordan. Environ Sci Pollut Res Int 9:1-8. https://doi.org/10.1007/s11356-020-12338-y

Borges JT, Nakada LYK, Maniero MG, Guimarães JR (2021 Feb) SARS-CoV-2: a systematic review of indoor air sampling for virus detection. Environ Sci Pollut Res Int 25:1-14. https://doi.org/10. 1007/s11356-021-13001-w

Borisova T, Komisarenko S (2020 Oct) Air pollution particulate matter as a potential carrier of SARS-CoV-2 to the nervous system and/or neurological symptom enhancer: arguments in favor. Environ Sci Pollut Res Int 13:1-7. https://doi.org/10.1007/s11356-020-11183-3

El Aferni A, Guettari M, Tajouri T (2020 Oct) Mathematical model of BoltzmannÕs sigmoidal equation applicable to the spreading of the Coronavirus (Covid-19) waves. Environ Sci Pollut Res Int 15:1-9. https://doi.org/10.1007/s11356-020-11188-y

El-Sayed A, Aleya L, Kamel M (2021 Feb) COVID-19: A new emerging respiratory disease from the neurological perspective. Environ Sci Pollut Res Int 15:1-15. https://doi.org/10.1007/s11356-021-129699

Guettari M, Gharbi I, Hamza S (2020 Oct) UVC disinfection robot. Environ Sci Pollut Res Int 14:1-6. https://doi.org/10.1007/s11356020-11184-2

Kalan ME, Ghobadi H, Taleb ZB, Adham D, Cobb CO, Ward KD, Behaleh R, Fazlzadeh M (2020 Oct) COVID-19 and beliefs about tobacco use: an online cross-sectional study in Iran. Environ Sci Pollut Res Int 7:1-9. https://doi.org/10.1007/s11356-020-11038-x

Kumar R, Sharma A, Srivastava JK, Siddiqui MH, Uddin MS, Aleya L (2021 Jan) Hydroxychloroquine in COVID-19: therapeutic promises, current status and environmental implications. Environ Sci Pollut Res Int 15:1-14. https://doi.org/10.1007/s11356-020-122001

Malki Z, Atlam ES, Ewis A, Dagnew G, Ghoneim OA, Mohamed AA, Abdel-Daim MM, Gad I (2021 Apr) The COVID-19 pandemic: prediction study based on machine learning models. Environ Sci Pollut Res Int 10:1-11. https://doi.org/10.1007/s11356-021-138247

Raza A, Ali Q, Hussain T (2020a Oct) Role of knowledge, behavior, norms, and e-guidelines in controlling the spread of COVID-19: evidence from Pakistan. Environ Sci Pollut Res Int 4:1-17. https:// doi.org/10.1007/s11356-020-10931-9

Raza A, Khan MTI, Ali Q, Hussain T, Narjis S (2020b Oct) Association between meteorological indicators and covid-19 pandemic in Pakistan. Environ Sci Pollut Res Int 14:1-16. https://doi.org/10. 1007/s11356-020-11203-2 
Sahoo MM (2021 Feb) Significance between air pollutants, meteorological factors and COVID-19 infections: probable evidences in India. Environ Sci Pollut Res Int 27:1-22. https://doi.org/10.1007/s11356021-12709-Z

Tabish M, Khatoon A, Alkahtani S, Alkahtane A, Alghamdi J, Ahmed SA, Mir SS, Albasher G, Almeer R, Al-Sultan NK, Aljarba NH, AlQahtani WS, AL-Zharani M, Nayak AK, Hasnain MS (2020) Approaches for prevention and environmental management of novel COVID-19. Environ Sci Pollut Res Int:1-11. https://doi.org/10. 1007/s11356-020-10640-3

Yin H, Sun T, Yao L, Jiao Y, Ma L, Lin L, Graff JC, Aleya L, Postlethwaite A, Gu W, Chen H (2021 Jan) Association between population density and infection rate suggests the importance of social distancing and travel restriction in reducing the COVID-19 pandemic. Environ Sci Pollut Res Int 13:1-7. https://doi.org/10. 1007/s11356-021-12364-4

Publisher's note Springer Nature remains neutral with regard to jurisdictional claims in published maps and institutional affiliations.

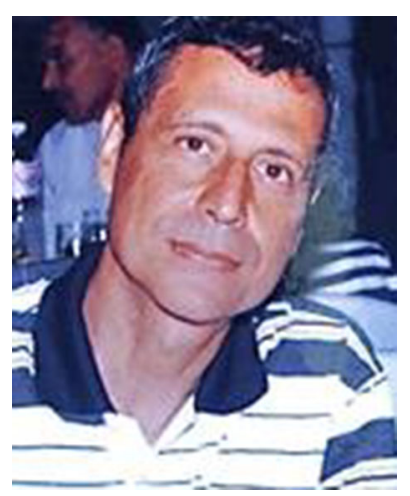

Dr. Lotfi Aleya is a Exceptional Class Professor of Biology, teaching at both the Faculty of Science and Faculty of Medicine, Besançon, France. He obtained his Ph. D. from Blaise-Pascal University (National Center for Scientific Research, France). He published over than 300 papers on experimental studies and review articles at many peerreviewed journals related to Microbiology, Protistology, Medecine, Environmental $\mathrm{Neurology,} \mathrm{Harmful}$ Microrganisms, Ecotoxicology. He is Associate Editor of Science of the Total Environment, Editor of Environmental Science and Pollution Research, Editor of Sustainability, Associate Editor of Critical Reviews in Environmental Science and Technology, Review Editor of Inflammation Pharmacology, Advisory member of Harmful Algae, Guest Editor of Oxidative Medecine and Cellular Longevity,.... He pilots cooperative projects in France and other countries, engaging private sector companies and public sector research centers associated with his Chair. He published more than 50 books and chapters and supervised $33 \mathrm{Ph}$. D thesis.

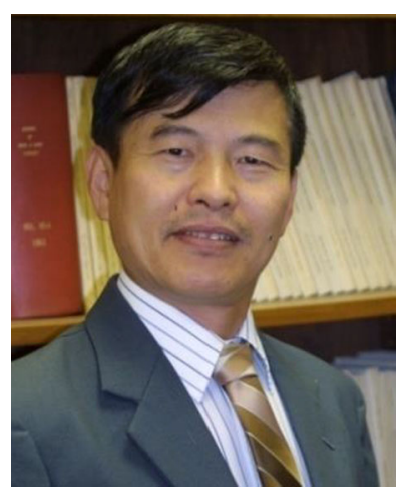

Dr. Weikuan Gu obtained his $\mathrm{PhD}$ from Cornell University. $\mathrm{He}$ is tenured and a full Professor in the Department of Orthopaedic Surgery and Biomedical Engineering. He is the Director of translational Research in the department and the director for the DNA Discovery Core of the UTHSC Center of Genomics and Bioinformatics. Dr. Gu has a joint appointment with Graduate Faculty in Department of Pathology, Cancer Research Center, Division of Connective Tissue Diseases, in the College of Medicine, and Department of Pharmaceutics Sciences, in the College of Pharmacy. He has published more than 130 peer reviewed full publications, more than 100 abstracts/ presentations/invited lectures, and 10 book chapters. He edited a book named "Gene Discovery for Disease Models" which is published in early 2011. He also holds a patent for identification of suitable patients for oral induced tolerance to arthritis and scleroderma. He served as a member of editor board for the Case report (during 2008 and 2010), Chinese Journal of Endemiology, and Annals of Musculoskeletal Disorders. He serves as reviewers for 21 medical science journals. He coordinated many international education and research collaborative activities. He trained and/or coordinated the training of more than 100 people, including post-doctoral researchers, graduate students, summer students, and visiting scientists.

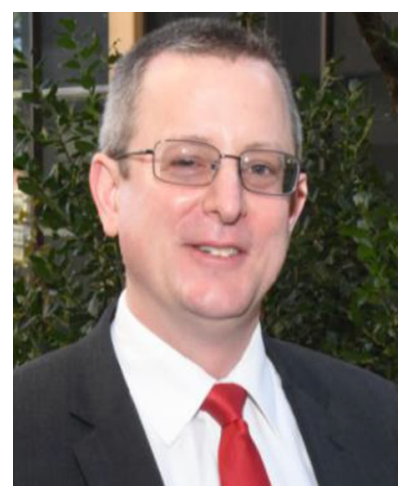

Scott Howard After training in internal medicine, pediatrics, and epidemiology at the University of Tennessee and completing a hematology/oncology fellowship at St. Jude Children's Research Hospital, Scott worked for 14 years as a faculty member treating children with leukemia and lymphoma and conducting research in oncology supportive care, international oncology, and healthcare informatics. He served as the Medical Information Officer and chief informaticist for the international outreach programs of St. Jude, then left St. Jude in 2014 to found Resonance (www.AmplifyingHealth.com), a non-profit providing informatics, consulting, education, and global health expertise to improve care and outcomes in low- and middle-income countries (LMIC). He returned to academia in 2017 as a professor at the University of Tennessee Health Science Center (www.UTHSC.edu), and focused on clinical research, global health, and educational programs for healthcare providers in all continents, and consulting for the World Health Organization to launch the Global Initiative for Childhood Cancer, with special focus on access to essential medicines and workforce capacity building. He is especially focused on improving clinical care and applying genomics, pharmacogenomics, big data, information systems, and point-of-care informatics tools to improve patient care and outcomes. His research interests also include global access to essential medications, cancer supportive care, epidemiology, and developing cancer programs in low- and middle-income countries.

Scott has authored or co-authored over 200 scientific papers, 20 book chapters, and one book. He conducts translational and clinical research with local and international collaborators, serves as the Secretary General 